\title{
Evaluation of the Hybrid Pedagogic Method in Students' Progression in Learning Using Neural Network Modelling and Prediction
}

\author{
Ethan Lau \\ Queen Mary University of London \\ Kok Keong Chai \\ Queen Mary University of London \\ Vindya Wijeratne \\ Queen Mary University of London \\ Gokop Longinus Goteng \\ Queen Mary University of London
}

The COVID-19 pandemic has changed dramatically the way how universities ensure the continuous and sustainable way of educating students. This paper presents the evaluation of the hybrid pedagogic methods in students' progression in Learning using neural network (NN) modelling and prediction. The hybrid pedagogic approach is based on the revised Bloom's taxonomy in combination with the flipped classroom, asynchronous and cognitive learning approach. Educational data of labs and class test scores, as well as students' total engagement and attendance metrics for the programming module are considered in this study. Conventional statistical evaluations are performed to evaluate students' progression in learning. The NN is further modelled with six input variables, two layers of hidden neurons, and one output layer. Levenberg-Marquardt algorithm is employed as the back propagation training rule. The performance of neural network model is evaluated through the error performance, regression, and error histogram. Overall, the NN model presents how the hybrid pedagogic method in this case has successfully quantified students' progression in learning throughout the COVID-19 period.

Keywords: pedagogic approach, blended learning, statistical evaluations, neural network

\section{INTRODUCTION}

Over the past months, the daily-routine life for nationals have been hugely affected by the coronavirus COVID-19 crisis. Therefore, the worldwide educational sectors should focus on the long-term continuity of education deliveries to guarantee a resilient education for equitable and sustainable development (United Nations, 2020). Consequently, many universities around the world are shifted to virtual online teaching, learning and assessments. However, this requires collective efforts by leveraging the pedagogic approach to enhance the online learning platforms while ensuring the vision and strategy of the universities. Many 
pedagogic methods have been put forward but the methodology to assess and predict students' progression of learning is extremely limited at the present pandemic situations. The conventional questionnaire and feedback surveys from students are not sufficiently enough to evaluate and quantify students' overall learning progression and engagement.

For this reason, this paper presents the neural network (NN) modelling and predicting students' progression in learning through the hybrid pedagogic method. We present how the computer software programming module considered in this study have successfully implemented the hybrid pedagogic method. The hybrid pedagogic approach is based on the revised Bloom's taxonomy in combination with the flipped classroom, asynchronous and cognitive learning, while maximising students' learning experiences. By effectively evaluating students' engagement in the online learning platform this will greatly help to assess the effectiveness of the hybrid pedagogy approach. This is done by evaluating students learning behaviour through their level of engagements in the module. The data information about students' engagement metrics are obtained through the lecture recording software. Apart from that, the assessments such as the lab and the class test results are adopted for statistical evaluations and the latter NN development. This further presents an exploratory NN modelling, and analyses students' progressing in learning and serves as a dominant educational quality tool to mitigate those disparities that hamper the education quality.

The organisation of the paper is as follows: Section 2 presents the reviews of pedagogic methods, the $\mathrm{NN}$ and the summary, Section 3 presents the methodology of map the hybrid pedagogic approach into NN modelling, Section 4 presents statistical evaluations, NN configuration settings and the performance, Section 5 concludes the findings.

\section{OVERVIEW OF THE PEDAGOGIC METHODS}

The traditional pedagogic format includes the behaviourism, constructivism, social constructivism and liberationism. Bloom's taxonomy is one of the most well-known hierarchical-based pedagogical tool to complement the strategies of educational objectives (Granello, 2001). Each of the hierarchy contains a domain of intellectual behaviour that stratifies teaching, learning and assessment activities. It has been well used in software engineering and computer science-specific learning domains (Fuller et al., 2007; Britto and Usman, 2015; Peter et al., 2016) and has helped in improving the assessment methods in the domain of science, technology, engineering, and mathematics (STEM).

Ever since the pandemic began, the pedagogy in the educational field is changing rapidly, where face to face delivery is no longer the best option to interact with students. Therefore, there is a need to create a balance of joint teaching elements in in-class and online while meeting students' learning outcomes. Together with the evolvement of digital academics, universities are looking for the approach to ensure the continuous engagement and active learning among students. Several recent papers paid a huge emphasis on Bloom's revised taxonomy to ensure adaptive online-based learning module in responding to the pandemic (Qadir and al-Furqaha, 2020; Sheth et al., 2020). Therefore, to accommodate the needs of students' learning needs during and beyond COVID-19, the revised Bloom's taxonomy is proposed in this paper that brings together the hybrid learning-based approach - flipped classroom, asynchronous and cognitive learning.

The revised Bloom's taxonomy is presented in Figure 1 where the hybrid pedagogical approach is implemented. This serves as a strategy to avoid just having to deliver modules but being able to prepare students to have conceptual understanding to solve problems and represent situations (Joffrion, 2005). The flipped classroom is a type of blended learning where students at first are being introduced the content at off-campus (pre-classroom activities) and work together with lecturers to solve the content during the live lectures (virtual classroom activities). Existing benefits of using flipped classroom have already been established, where one of the examples of case study students with flipped classroom $(\mathrm{N}=24)$ outperformed in exams compared with the traditional ( $\mathrm{N}=19)$ (Peterson, 2016).

In addition, the hybrid approach is part of the strategy to activate students' cognitive-based prior knowledge. Activating prior cognitive knowledge brought more beneficial effect on knowledge and comprehension understanding to enable students to learn actively (Kostons and Werf, 2015), and thus 
facilitates the conceptual understanding of science and engineering principles and increases students' selfregulated responsibility in participating the lecture. Furthermore, the flipped classroom implemented with cognitive-based learning could lead to increased academic performance (Foldnes, 2016).

Asynchronous learning is another prevalent pedagogical strategy where it is used in "Anytime, Anywhere" basis that includes instructor-led online face-to-face meetings and chats, recorded lectures, and tutorials (Wu et al., 2008). Asynchronous learning method has been well received, where it creates a great potential for enhancing cognitive learning progress and the resultant academic performance among students (Michalsky and Zion, 2007).

\section{FIGURE 1 \\ THE REVISED BLOOM'S TAXONOMY}

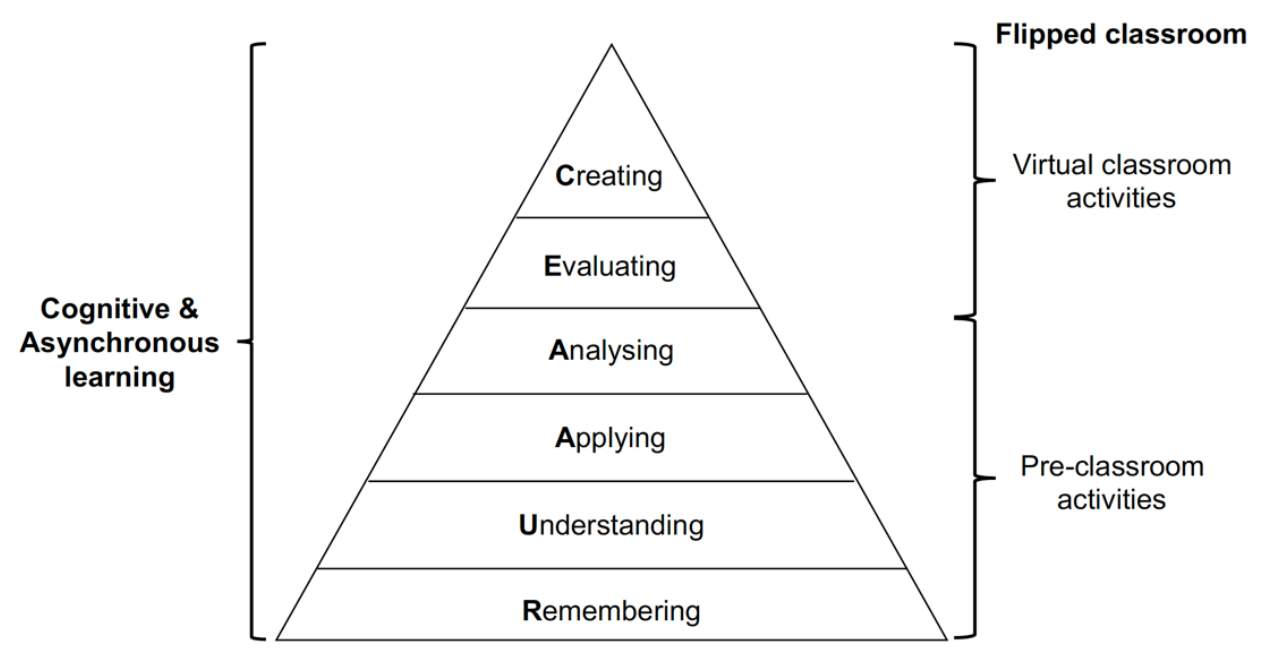

In summary, the hybrid pedagogical approach is applied to improve the existing Bloom's taxonomy in responding to students' learning needs. This is to ensure students have more spaces for them to elicit the conceptual information when watching the recorded videos, and to further allow more space for students' talk time and understanding on the topic in the virtual face-to-face meetings. Such mechanism ensures more learning spaces for students and not solely depending on the traditional lecturer-centred rule.

\section{Neural Network}

With the expansion of the education system together with the restriction in COVID-19, various pedagogy methods are not being evaluated analytically that can be combined with machine learning techniques for educational research purposes. Machine learning techniques leverage the pedagogical methods that detect at-risks students earlier and to further mitigate negative consequences from occurring (Macarini et al., 2019). Additionally, machine learning techniques improve the accuracy of prediction by collectively classifying the low-engagement students, intervening their learning behaviour and improve their learning engagements (Hussain et al., 2018).

Therefore, this paper implements a neural network (NN) model that is capable of machine learning by collecting students' information and further predicting students' progression in learning based on their learning engagements. $\mathrm{NN}$ is formed through a collection of artificial neurons that resemble the connection geometry of neurons in human brains to execute a task with improved performance through learning, training, and continuous improvement (Vandamme et al., 2007; Kardan et al., 2013). The most common learning rule of $\mathrm{NN}$ is back-propagation (BP), which is a supervised learning approach and can be used for training the deep neural networks. The weight of the neurons is adjusted using the BP accordingly. This enables the NN to learn from the training process through the calculated errors from the BP. 
Remarkably, NN approach is used widely in the educational field. It has been used to not only to accurately predict final grades of students, but also to improve the better understanding of the educational process and evaluation (Kardan et al., 2013; Isljamovic and Suknovic, 2014; Okubo et al., 2017).

Overall, NN has the ability to predict and classify any data with subjective accuracy. This is important to examine the substantial factors that affect the students' progression in learning through the proposed pedagogic approach. The NN model would serve as a framework and an important decision-making tool that evaluates the reformed pedagogical approaches towards students' progression in learning and their performance.

\section{METHODOLOGY}

This section presents the methodology of implementing the hybrid pedagogic approach in the revised Bloom's taxonomy, acquiring educational data, statistical testing for NN modelling, and evaluating the performance of NN.

\section{Overview of the Module}

The computer software programming module is considered in this study. The module aims to introduce different software service platforms and how it is used to enable heterogeneous systems to work together within a distributed cloud system. At the end of this module, students will have acquired the knowledge of the different types of software service platforms available, design and implement client-server or web-based applications. A total of 181 Year 3 undergraduate students are currently enrolled to the module. Apart from the delivery of live and recorded lectures (which will be explained in the next section) as well as the tutorials, the module's lab assessment is based on four sessions of progressive-based lab exercises covered by four lab sessions in 4th, 6th, 8th and 9th of the teaching weeks. Lab task difficulties are increased when students progress themselves until the final session of the labs. Students need to submit all the lab tasks through the online Moodle learning portal accordingly. After the completion of lab exercises, students are then needed to take the class test (Week 12th) that aims to evaluate students' performance throughout the module learning. All lab assessments and class tests are performed online while ensuring the important aspect of the UK Quality Code for Higher Education in achieving the intended learning outcomes.

\section{The Hybrid Pedagogic Approach}

The revised Bloom's taxonomy is proposed in this paper which consists of the hybrid pedagogical approach - flipped classroom, asynchronous and cognitive learning. An example of asynchronous learning based on anytime, anywhere basis is presented in Table 1, where students are updated about the asynchronous lecture time and information on the Moodle. This is to avoid one-way limited communication flow from the existing Bloom's taxonomy. It is important to note that the hybrid strategy proposed in this paper does not improve the Bloom's taxonomy but takes students to a higher cognitive level. Microsoft Teams is the core lecturing and communication platform for both lectures and tutorials, apart from the Moodle portal to enable students to access the additional resources related. The module team channel is created in Microsoft Teams to allow students studying the computer software programming module to post any questions related to their learnings.

TABLE 1

ASYNCHRONOUS LEARNING TIMETABLE

\begin{tabular}{ccccc}
\hline Session & Week 1 & Week 2 & Week 4 & Week 5 \\
\hline Thursday, 8:35 -9:20 am & Live & Recorded & Live & Recorded \\
Thursday, 9:25 - 10:10 am & Live & Live & Live & Recorded \\
\hline
\end{tabular}

Flipped classroom, on the other hand, is implemented as part of the blending learning approach that comes with pre-recorded video sessions. The technique allows pre-classroom activities where students need 
to watch the pre-recorded video either on/off campus, and then continuing the learning by attending the live lectures to solve the problems. This is done via virtual face-to-face classroom activities.

To improve students' learning and comprehension especially in dealing with the pandemic, all the tutorials, online quizzes and lab tasks are carefully constructed based on students' previous knowledge in fundamental programming concepts (e.g. Java Programming). Such serves as a prerequisite for students to undertake the computer software programming module. Together with recorded video watching, this serves as a pre-cognitive domain to activate students' prior knowledge, and to further apply the cognitive knowledge that facilitates the conceptual understanding of the module during the live lecture session. This increases students' self-regulated responsibility in participating the lecture, and thereby improving the interactions, engagements and their resultant learning behaviour in the module.

\section{Data Collection}

No questionnaire or feedback surveys are conducted in this case. Instead, the evaluations are performed using the data analytics and are collected from the lecture recording software, lab assessments, and the class test. Additionally, the assessments are performed solely to the Year 3 cohort undertaking the computer software programming module. In terms of students' behaviour and progression in learning, the dataset information is available and extracted using the Echo360 lecture recoding software. The Echo360 software provides the course analytics metrics (e.g. engagement, slide views, video views, polling responses, attendances) that rolls up the data information (Echo360, 2020) for each student enrolled in the course. In this paper the metrics Total engagement and Attendance are extracted as the metrics are the important factors that influence students' learning and progression behaviour, and the resultant performance. The Total Engagement in this case is the cumulative total of data points such as Video Views, Q\&A Entries, Polling Responses (polling questions that were answered correctly) and Note Events (total number of distinctive notes student have taken). Attendance is where students entered the online classroom during the class time.

\section{Statistical Testing}

Prior to the NN modelling, it is required to observe the relationship between the students' progression and learning behaviour that further indicate the level of engagement in the module, lab assessments and their class test scores. Statistical testing is performed initially in this study using multiple regression analysis. Multiple regression analysis is used to evaluate whether a dependent variable could be predicted based on independent variables. The significant difference is set at $\alpha=0.05$. Pearson correlation coefficients are calculated to measure the linear relationships of four lab assessment scores, the students' engagement metrics (Total engagement and Attendance), and the resultant class test score. Those correlated variables are added to the input neurons for the NN modelling.

\section{Neural Network Modelling}

The NN predicts students' progression in learning and engagement through input observations of lab assessments, the metrics of students' engagement and the class test scores. Such scheme is performed based on the supervised machine learning. The NN in this paper is formulated using the earlier works (Zhang et al., 2003; Vandamme et al., 2007; Rashid and Ahmad, 2016; Lau et al., 2019). This paper does not compare the effectiveness of NN performance with other machine learning techniques where it is intended to focus on the evaluation of the hybrid pedagogic approach in students' progressing in learning using NN.

By conventional definition, the NN mathematical model function can simply be formulated as:

$$
\tilde{Y}=f(\tilde{X}, \widetilde{W}) \text {, }
$$

where $\tilde{Y}$ and $\tilde{X}$ are the output and input vectors. $\widetilde{W}$ is a weight vector of connections within the NN. The input layer collects data with feature sets. The input values are fed to the hidden layer. The output values 
of $j$ th neuron $y_{j}$ of vector $\tilde{Y}$ are calculated through the weighted sum of input elements $x$ and $w$, where $w$ is updated recursively.

$y_{j}=\theta\left(\sum_{i=1}^{N_{i}} w_{i j} x_{i}\right)$.

The $\theta$ is the activation function (transfer function), $N_{i}$ is the total number of $i$ th connection lines to the $j$ th neuron and $x_{i}$ is the output value from the previous layer of $i-l$ th neuron. The hyperbolic tangent function is used as the activation function $(\theta)$ to transfer the value of weighted sum of inputs to the output layer.

The activated node for the next input layer is:

$x_{j}=\theta\left(y_{j}\right)$

For training, both inputs and outputs parameters are supplied to the NN model. BP is used as the learning rule for the NN model by altering the weights of neurons $w_{i j}$ accordingly through the errors computed recursively that further produces desired outputs. The error function $(E)$ is calculated as:

$E=\frac{1}{2} \sum_{j}^{N_{j}}\left(y_{j}-t_{j}\right)^{2}$

where $t_{j}$ is the target value for neuron $i$ in the output layer and $N_{j}$ is the total number of output neurons.

The BP-based Levenberg-Marquardt optimisation algorithm is applied in the NN training. It speeds up the optimisation to an optimal solution with convergency by effectively solving the training algorithms for non-linear problems (Yu and Wilamowski, 2011; Wilson and Mantooth, 2013). The Levenberg-Marquardt is a hybrid-based training method using the steepest descent (gradient descent) and Gauss-Newton method. The algorithm applies the approximation to Hessian Matrix that is similar to the Gauss-Newton method (Yu and Wilamowski, 2011; Math- Works, 2019):

$w_{i j+1}=w_{i j}-\left[J^{\prime} J+\zeta I\right]^{-1} J^{\prime} \omega_{k}$,

where $J$ denotes the Jacobian matrix, $\omega_{k}$ is the error in the network (MathWorks, 2019), $w_{i j}$ is the current weight and $w_{i j}$ is the updated weight, $\zeta$ is the damping factor.

When $\zeta$ is small, the Levenberg-Marquardt training algorithm in Eq. 5 applies the Gauss-Newton method and becomes gradient descent algorithm when $\zeta$ is large. IN general, $\zeta$ is altered at every iteration and also acted to switch between those two algorithms as the training model.

The number of neuron in the output layer is the resultant decision prediction of the problem (Özçelik and Hardalaç, 2011). Finally, the output layer is the vector $\tilde{Y}$ that consists of the collection of predicted class test.

\section{NN Performance Evaluation Criteria}

To evaluate the NN performance, this paper computes the Mean Square Error (MSE), regression analysis, and error histogram that mitigates the arising of over-fitting issues to ensure the appropriateness of NN performance. A well-trained NN model should have low MSE value, which means that the predicted outputs converge closely to the target outputs (tij) (provided that no over-fitting issues occurred). MSE is calculated as:

$M S E=\frac{1}{N_{i} N_{j}} \sum_{j=1}^{N_{j}} \sum_{i=1}^{N_{i}}\left(y_{i j}-t_{i j}\right)^{2}$.

To avoid over-fitting of the trained network when the MSE value is low, a regression is performed along with computed R-value which demonstrates the goodness of fitting between the predicted and the desired outputs. Such ensures that the NN only works well in the training validation and testing phase. The 
R-value is useful in examining the end performance of fitting. If poor fitting (low R-value) is obtained, further trainings are required with modification of hidden layers and neurons.

The error histogram is also used to measure the NN performance. With error histogram distribution plot, most errors are occurred near zero. The error is simply the difference between the targeted outputs tij and the predicted outputs yij as formulated in Eq. 4.

\section{RESULTS}

This section presents the findings, the configuration of $\mathrm{NN}$, the prediction and the verification of $\mathrm{NN}$ performance for 181 Year-3 undergraduate students. This is based on the obtained datasets of lab assessments and class test scores, as well as the information about students' engagement and the attendance metric from the Echo360 software.

In summary the average class test score is $78.19 \pm 10.10$ compared with $81.02 \pm 11.18$ for the previous academic year, which stays consistent when the teaching model was very different.

\section{Statistical Evaluations}

Multiple regression analysis is used to give a better indication whether the Class Test (the dependent variable) could be predicted based on the independent variables - Lab scores, students' Total Engagement and the Attendance metrics. In this paper, we are focusing on demonstrating the three main outputs for the multiple regression analysis as such verifies the need for latter NN model simulation and analysis. The outputs are the regression summary, Analysis of Variance (ANOVA) and statistical significance analysis of the independent variables.

Table 2 provides the R, R2, adjusted R2 and the standard error of the estimates. The results show a marginally good quality of the prediction $\mathrm{R}$ value (the class test) of 0.621 . Additionally, the adjusted R2 value of 0.569 indicates that independent variables contribute to $56.9 \%$ of the variability of the dependent variable.

TABLE 2

SUMMARY OF REGRESSION MODEL

$\begin{array}{cc}\text { Multiple } \boldsymbol{R} & 0.621 \\ \boldsymbol{R}^{2} & 0.599 \\ \text { Adjusted } \boldsymbol{R}^{2} & 0.569 \\ \text { Standard Error } & 7.132\end{array}$

The ANOVA tests the goodness of fits for the overall multiple regression analysis. The result is shown in Table 3. As $\alpha<0.05$, the model is a good fit of the data for the NN model.

TABLE 3

ANOVA RESULT

\begin{tabular}{cccccc}
\hline Source & Sum of square & d.f. & Mean square & F-cal & $\boldsymbol{\alpha}$ \\
\hline Regression & 608.67 & 6 & 101.44 & 1.563 & 0.002 \\
Residual & 11422.2 & 176 & 64.90 & & \\
Total & 12030.87 & 182 & & & \\
\hline
\end{tabular}

The statistical significance for each of the independent variables is shown Table 4 . As $\alpha<0.05$, all the independent variables are statistically significant to the dependent variable (the class test). 
TABLE 4

STATISTICAL SIGNIFICANCE OF THE INDEPENDENT VARIABLES

\begin{tabular}{ccccc}
\hline Model & Coefficients & Standard Error & T Stat & $\boldsymbol{\alpha}$ \\
\hline (Intercept) & 87.83 & 6.390 & 13.745 & 0.001 \\
Lab 1 & -0.243 & 0.075 & -3.24 & 0.000 \\
Lab 2 & -0.198 & 0.052 & -3.808 & 0.000 \\
Lab 3 & -0.423 & 0.032 & 13.218 & 0.001 \\
Lab 4 & -0.165 & 0.059 & -2.796 & 0.004 \\
Total Engagement & -0.118 & 0.032 & -3.667 & 0.000 \\
Attendance & 13.116 & 1.344 & 9.756 & 0.000 \\
\hline
\end{tabular}

Pearson correlation coefficients are calculated to examine the degree of closest relationship among the four Lab scores, the students' engagement metrics (Total engagement and Attendance), and the resultant class test score. The correlation coefficients between lab scores, total engagement, attendance, and the class test score are shown in Table 5, with ascending order: Total Engagement, Lab 1, Lab 2, Lab 3, Lab 4 and Attendance. The correlated variables applied into the NN modelling as input neurons.

For the Person Correlation test, surprisingly, the correlation coefficient value increases as students progress themselves from Lab 1 to Lab 4. Apart from that, the attendance metric is with the highest relationship with the class test score. This shows that attending the live and recorded lecture, as well as completing the lab assessments are important to score better in the class test. This is in line with the explanation where students can solve programming questions and perform well with cognitive levels as high as application and analysis in online learning activities (Othman and Zain, 2015).

TABLE 5

CORRELATION COEFFICIENT OF FOUR LAB ASSESSMENTS, TOTAL ENGAGEMENT AND ATTENDANCE, AND THE CLASS TEST SCORE

\begin{tabular}{cccccccc}
\hline Model & Lab 1 & Lab 2 & Lab 3 & Lab 4 & $\begin{array}{c}\text { Total } \\
\text { Engagement }\end{array}$ & Attendance & Class Test \\
\hline Lab 1 & 1 & & & & & & \\
Lab 2 & 0.84 & 1 & & & & & \\
Lab 3 & 0.70 & 0.70 & 1 & & & & \\
Lab 4 & 0.85 & 0.84 & 0.80 & 1 & & & \\
Total & 0.31 & 0.30 & 0.28 & 0.31 & 1 & 1 & \\
Engagement & & & & & & \\
Attendance & 0.34 & 0.31 & 0.28 & 0.32 & 0.92 & 0.42 & 1 \\
Class Test & 0.36 & 0.37 & 0.40 & 0.41 & 0.36 & \\
\hline
\end{tabular}

\section{NN Configuration Settings}

MathWorks MATLAB software is used to model and evaluate the NN. The input layer consists of six variables containing all the four lab assessment scores and the metrics include the Total Engagement and the Attendance. The NN model contains two hidden layers 30 hidden neurons in each layer. Such configuration provides the optimal NN simulation outcome. The hidden layer is finally fed into a single output neuron that represents the decision of the variable - the class test prediction.

The hyperbolic tangent is used as the activation function. The dataset is rationed and divided for training, validation, and testing purposes. Data samples of 181 students are randomly mixed and 0.7 of the mixed samples are used for training, 0.15 for validation, and finally the 0.15 ratio for testing.

During the training and learning phase, Levenberg-Marquardt algorithm in Eq. 5 determines the optimal weights for the next input layer. The damping factor $\zeta$ is set as 0.001 and the training epoch is as 2,000 . The 
$\mathrm{NN}$ training runs continuously and terminates when the computed error values do not decrease for six iterations consecutively during the validation process to protect $\mathrm{NN}$ model from over-training.

\section{NN Simulation Results}

After the completion of $\mathrm{NN}$ training, testing and validations, the NN performance with MSE $\approx 58.74$ or $6.9 \%(<10 \%)$ indicates the sufficiently good performance of NN. Regression plots of NN performance are shown in Figure 2. For a good data fitting, the predicted outputs yij should match closely with the target outputs tij. In this case, the regression plot results prove marginally good fits with an overall R-value of 0.595 .

The error histogram evaluates the error distributions of the NN predictions. The error distribution is shown in Figure 3 where errors are occurred near zeroth point (horizontal axis) and are gradually decreasing when moving away from zeroth point. This proves positive aspects of $\mathrm{NN}$ prediction with adequate error distributions as shown in Figure 3.

FIGURE 2

\section{REGRESSION PLOT FOR NN MODEL: TOP LEFT: TRAINING DATA; BOTTOM LEFT: TEST DATA; TOP RIGHT: VALIDATION DATA; BOTTOM RIGHT: RESULTANT REGRESSIONS}
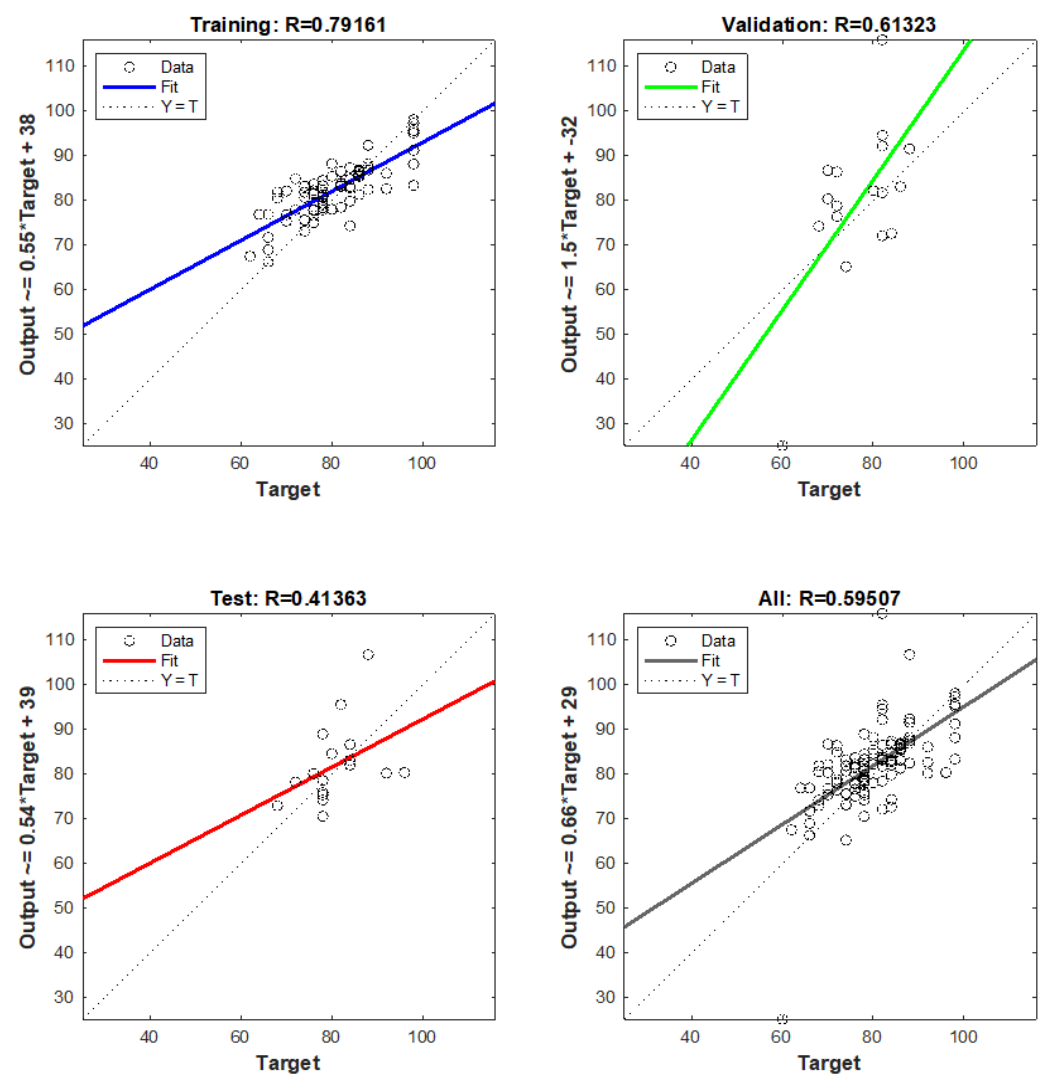


\section{FIGURE 3 \\ ERROR HISTOGRAM PLOT}

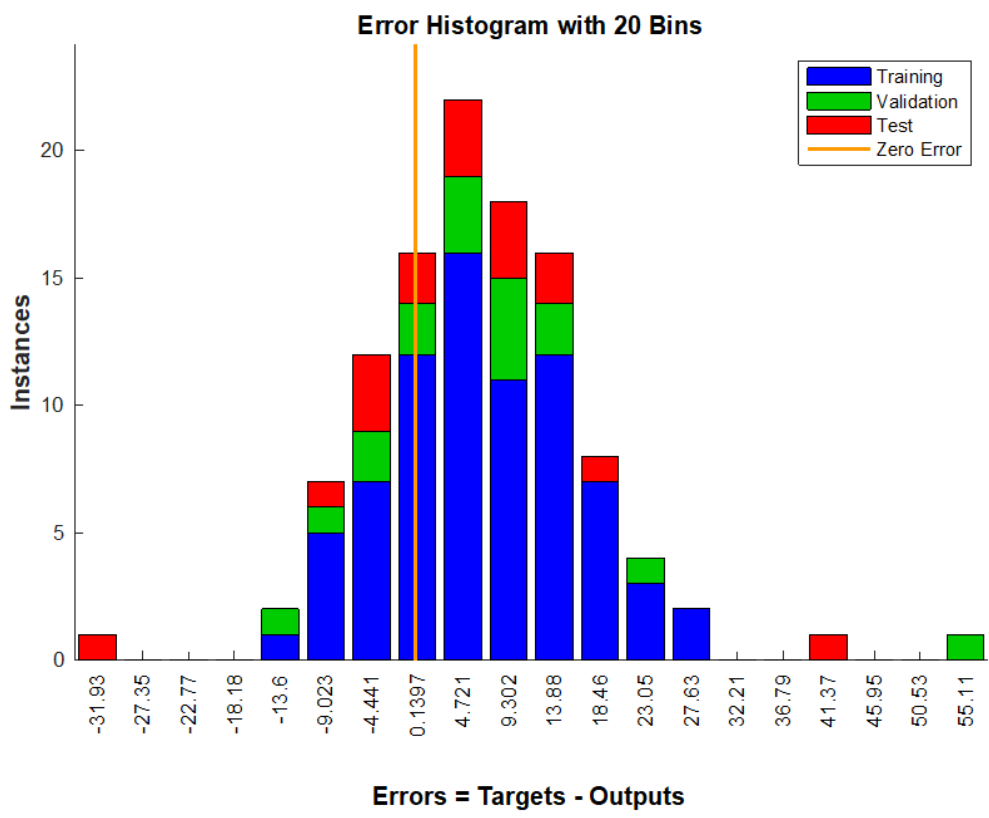

\section{CONCLUSIONS}

In this paper NN is used to model, evaluate, and predict students' progression in learning through the employed hybrid pedagogic approach - revised Bloom's taxonomy in combination of flipped classroom, asynchronous and cognitive learning throughout the COVID-19 period. This is accomplished using available dataset about their lab assessment scores, class test and the information about their engagements and attendance metrics using the lecture recording software analytics.

The multiple regression result showed the statistically significance of all the independent variables to the dependent variable. Such evaluations verified the appropriateness of variables used for the latter NN modelling. The Person Correlation test also shows the order of importance on how the progression of students from Lab 1 to Lab 4 plus attending the live and recorded video lectures will affect their class test scores.

Overall, the good NN performance makes NN such a useful tool for education evaluation purposes. However, some present limitations of this study are the low sample size of the dataset obtained. Yet, it is still strongly believed that education modelling settings using NN provides an instrumental contribution in evaluating students' academic performance. The future NN model should incorporate other attributes such as the lecturers' role, some other classroom activities such as quizzes, course feedbacks and formative assessments. We strongly believe that the proposed model is highly generalisable in fitting into other courses and thus leveraging the 'Smart' educational learning solutions. 


\section{REFERENCES}

Britto, R., \& Usman, M. (2015). Bloom's taxonomy in software engineering education: A systematic mapping study. In 2015 IEEE Frontiers in Education Conference (FIE) (pp. 1-8).

Echo360. (2020). Definitions of course analytics metrics. Retrieved November 11, 2020, from https://learn.echo360.com/hc/en-us/articles/360035037312

Foldnes, N. (2016). The flipped classroom and cooperative learning: Evidence from a randomised experiment. Active Learning in Higher Education, 17, 39-49.

Fuller, U., Johnson, C., Ahoniemi, T., Cukierman, D., Hernán-Losada, I., Jackova, J., . . Thompson, E. (2007). Developing a computer science-specific learning taxonomy. ACM SIGCSE Bulletin, $39(4), 152-170$.

Granello, D. (2001). Promoting cognitive complexity in graduate written work: Using bloom's taxonomy as a pedagogical tool to improve literature reviews. Counselor Education and Supervision, 40(4), 292-307.

Hussain, M., Zhu, W., Zhang, W., \& Abidi, S. (2018). Student engagement predictions in an e-learning system and their impact on student course assessment scores. Computational Intelligence and Neuroscience, 6, 1-21.

Isljamovic, S., \& Suknovic, M. (2014). Predicting students' academic performance using artificial neural network: A case study from faculty of organizational sciences. In ICEMST 2014: International Conference on Education in Mathematics, Science \& Technology (pp. 68-72). ISRES Publishing.

Joffrion, H. (2005). Conceptual and procedural understanding of algebra concepts in the middle grades. Master's thesis, Office of Graduate Studies of Texas A\&M University.

Kardan, A.A., Sadeghi, H., Ghidary, S.S., \& Sani, M.R.F. (2013). Prediction of student course selection in online higher education institutes using neural network. Computers \& Education, 65, 1-11.

Kostons, D., \& Werf, G. (2015). The effects of activating prior topic and metacognitive knowledge on text comprehension scores. British Journal of Educational Psychology, 85(3), 264-275.

Lau, E., Sun, L., \& Yang, Q. (2019). Modelling, prediction and classification of student academic performance using artificial neural networks. SN Applied Sciences, 1(982), 1-10.

Macarini, L., Cechinel, C., Machado, M., Ramos, V., \& Munoz, R. (2019). Predicting students' success in blended learning - evaluating different interactions inside learning management systems. Applied Sciences, 9, 1-23.

MathWorks. (2019). trainlm - Levenberg-Marquardt backpropagation. Retrieved June 17, 2019, from http://uk.mathworks.com/help/nnet/ref/trainlm.html

Michalsky, T., \& Zion, M. (2007). Developing students' metacognitive awareness in asynchronous learning networks in comparison to face-to-face discussion groups. Journal of Educational Computing Research, 36(4), 395-424.

Okubo, F., Yamashita, T., Shimada, A., \& Ogata, H. (2017). A neural network approach for students' performance prediction. In LAK17 - The Seventh International Learning Analytics \& Knowledge Conference (pp. 598-599). Association for Computing Machinery (ACM).

Othman, M., \& Zain, N. (2015). Online collaboration for programming: Assessing students' cognitive abilities. Turkish Online Journal of Distance Education-TOJDE, 16(4), 84-97.

Özçelik, S., \& Hardalaç, N. (2011). The statistical measurements and neural network analysis of the effect of musical education to musical hearing and sensing. Expert Systems With Applications, 38, 9517-9521.

Peter, D., Leth, T., \& Bent, T. (2016). Assessing problem-based learning in a software engineering curriculum using bloom's taxonomy and the IEEE software engineering body of knowledge. ACM Transactions on Computing Education, 16(3), 1-41.

Peterson, D. (2016). The flipped classroom improves student achievement and course satisfaction in a statistics course: A quasi-experimental study. Teaching of Psychology, 43, 10-15.

Qadir, J., \& al-Furqaha, A. (2020). A student primer on how to thrive in engineering education during and beyond COVID-19. Education Sciences, 10(9), 236-258. 
Rashid, T., \& Ahmad, H. (2016). Using neural network with particle swarm optimization. Computing Application Engineering Education, 24, 629-638.

Sheth, S., Ganesh, A., Nagendra, S., Kumar, K., Tejdeepika, R., Likhitha, C., . . Chand, P. (2020). Development of a mobile responsive online learning module on psychosocial and mental health issues related to COVID-19. Asian Journal of Psychiatry, 54, 236-258.

United Nations. (2020). Policy brief: Education during COVID-19 and beyond - August 2020. Technical report, United Nations.

Vandamme, J., Meskens, N., \& Superby, J. (2007). Predicting academic performance by data mining methods. Education Economics, 15(4), 405-419.

Wilson, P., \& Mantooth, H. (2013). Model-based engineering for complex electronic systems. Newnes.

Wu, D., Bieber, M., \& Hiltz, S. (2008). Engaging students with constructivist participatory examinations in asynchronous learning networks. Journal of Information Systems Education, 19(3), 321-330.

$\mathrm{Yu}, \mathrm{H} .$, \& Wilamowski, B. (2011). Levenberg Marquardt training industrial electronic handbook. Intelligent Systems (2nd edition, vol. 5). CRC Press,

Zhang, Q., Kuldip, C., \& Devabhaktuni, V. (2003). Artificial neural network for rf and microwave design - from theory to practice. IEEE Transactions on Microwave Theory and Techniques, 51(4), $1339-1350$.

70 Journal of Higher Education Theory and Practice Vol. 21(9) 2021 